\title{
Modeling the plasma plume of a hollow cathode
}

\author{
lain D. Boyd ${ }^{\text {a) }}$ \\ Department of Aerospace Engineering, University of Michigan, Ann Arbor, Michigan 48109-2140 \\ Mark W. Crofton \\ The Aerospace Corporation, Space Materials Laboratory, Los Angeles, California 90009-2957
}

(Received 21 January 2003; accepted 6 January 2004)

\begin{abstract}
In this study, a numerical model is developed to simulate the xenon plasma plume from a thermionic hollow cathode employing an orifice plate used for propellant ionization and beam neutralization in an electrostatic space propulsion system. The model uses a detailed fluid model to describe the electrons and a particle-based kinetic approach is used to model the heavy xenon ions and atoms. A number of key assumptions in terms of physical modeling and boundary conditions of the simulations are assessed through direct comparisons with experimental measurements. For two of the three cathode operating conditions considered, good agreement with the measured data is obtained. The third condition appears to lie in a different physical regime where elevated electron and ion temperatures and decreased transport coefficients are required in the simulation to provide agreement between the model and the measured data. (C) 2004 American Institute of Physics.
\end{abstract}

[DOI: $10.1063 / 1.1651333]$

\section{INTRODUCTION}

Thermionic hollow cathodes using an orifice plate are of general technological importance. One application is in electric thruster systems, where they serve as critical components providing an efficient source of electrons for both propellant ionization and beam neutralization tasks. As a thruster component hollow cathodes are much simpler to build and operate than the systems in which they are incorporated. Hollow cathodes will make attractive stand-alone ion thrusters if the ion emission current and device efficiency can become more comparable to existing ion thruster systems. The simplicity and small physical volume of hollow cathodes are attractive features for microsatellite or nanosatellite propulsion applications that may require the high specific impulse of ion propulsion. Behavior of hollow cathodes is complex, however, and poorly understood. An understanding of hollow cathode behavior is helpful to aid its development as a standalone ion thruster and as an optimized thruster component. Xenon hollow cathodes can produce single-point failures in ion engines and Hall effect thrusters, and they are an important factor regarding erosion of the screen grid and other components in ion engines. During operation at the high emission current required for high-power ion propulsion systems, the orifice, and any components in the plume erode rapidly. ${ }^{1}$ Ions of sufficient energy to cause significant erosion have been observed in plume experiments. ${ }^{2-4}$

Past measurements of the ion kinetic-energy distribution in the far-field plume of a hollow cathode have been performed with a retarding potential analyzer (RPA) or an energy analyzer, ${ }^{2-4}$ and more recently with a quadrupole mass spectrometer (QMS). ${ }^{5}$ The early results revealed that a very broad energy distribution exists in the high current regime. The data indicate that ions are abundantly formed with ener-

\footnotetext{
${ }^{a)}$ Electronic mail: iainboyd@umich.edu
}

gies as much as several times higher than the discharge potential.

The presence of abundant doubly charged ions was discovered through the QMS measurements. ${ }^{5}$ The higher charge states will contribute significantly to the erosive power of the hollow cathode plume. In addition, $\mathrm{Xe}^{2+}$ presence in abundance demonstrates a higher fraction of ionization than had previously been assumed. High fractional ionization may be a factor in the possible creation of a plasma potential that exceeds the anode voltage.

The mechanism by which the high-energy ions arise is not established, but two principal hypotheses have been put forward previously. One mechanism invokes the formation of a potential hill a short distance downstream from the orifice. ${ }^{3,6}$ Although consistent with some of the data, no clear understanding has emerged of the means by which a hill of sufficient height could be formed. An alternative mechanism has been postulated whereby the current density at the orifice (on the order of $10^{4} \mathrm{~A} \mathrm{~cm}^{-2}$ ) results in ion acceleration via a magnetohydrodynamic effect. ${ }^{2,7}$ A potential role may also exist for multiply charged ions in the production of energetic species with lower charge states. Unfortunately, RPA and energy analyzer devices do not distinguish between xenon ions with different charge states if their energy to charge ratios are identical. The energy to charge ratios will be the same for each charge state if the ions have been accelerated through the same potential difference, neglecting collision cross section variations and other effects.

A third hypothesis concerning the formation of highenergy ions has been applied to vacuum arc plasmas, where acceleration of ions also occurs in the direction away from the cathode and the energy to charge ratio can be much greater than the applied voltage. In this case, a gasdynamic model is invoked that describes ion acceleration as driven by pressure gradients and electron-ion friction. ${ }^{8,9}$ According to this approach, a small potential hump may exist as a conse- 


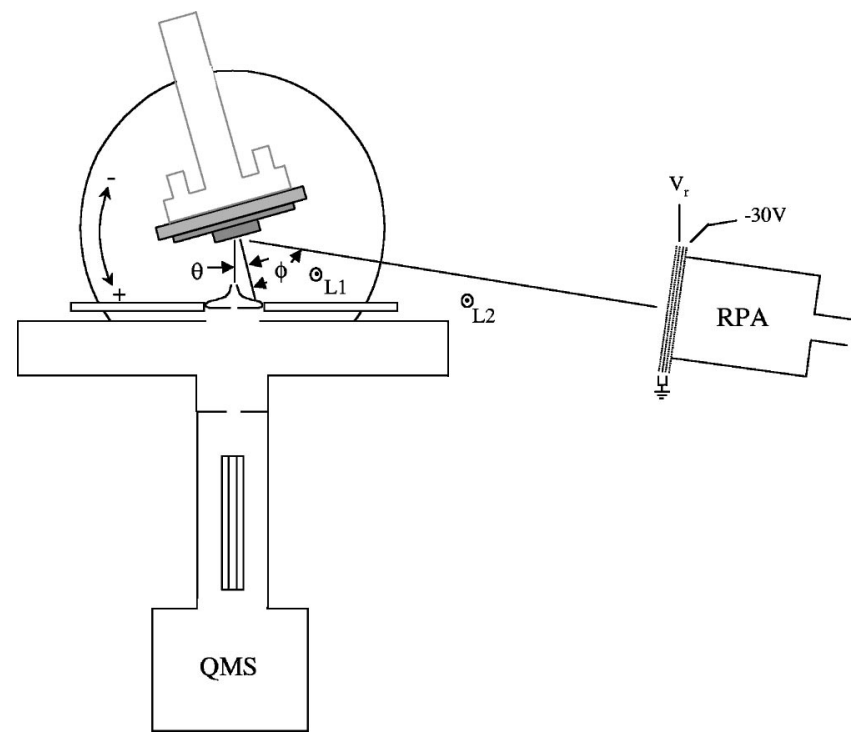

FIG. 1. Schematic of the experimental setup, top view.

quence of plasma acceleration rather than as its cause. The thermionic, orificed hollow cathode provides better stability and control than vacuum arcs for experimental studies, but relatively few measurements have been performed.

Spatially resolved experimental measurements of electric potential and the ion velocities near a hollow cathode orifice have previously been performed. ${ }^{10}$ A prominent potential hill was not found in either the Langmuir probe or laser-induced fluorescence measurements. The measured velocity distribution near the hollow cathode test articles depended strongly on the cathode type and/or operating point, including plume-mode versus spot-mode operation.

To try to help in obtaining a more complete understanding of these issues, in the present study, a computational model is developed to simulate the plasma plume emitted by a hollow cathode plume. The physical accuracy of the model is assessed through direct comparisons of the model results with experimental measurements taken in the plume of a xenon hollow cathode.

In the following, the hollow cathode and experimental investigation are described. Then, a detailed description of the numerical model is provided. Numerical results from the model are presented at three different operating points of the hollow cathode, and comparisons are made with the measured data. The article closes with some concluding remarks.

\section{HOLLOW CATHODE AND EXPERIMENT}

The hollow cathode studied was originally designed as the main cathode for xenon propellant ionization in the UK-10 electrostatic ion engine. The hollow cathode, shown schematically in Fig. 1, contains an impregnated tungsten dispenser, with an inside diameter of $1.0 \mathrm{~mm}$, an outer diameter of $2.8 \mathrm{~mm}$, and a length of $11 \mathrm{~mm}$, which acts as a chemical factory to release barium to the surface at an appropriate rate to achieve low work function and long life. The dispenser must be at $\sim 1000^{\circ} \mathrm{C}$ for the cathode to operate normally. The orifice of the hollow cathode, machined out of solid tantalum, is $0.2 \mathrm{~mm}$ in diameter, $1.0 \mathrm{~mm}$ long,

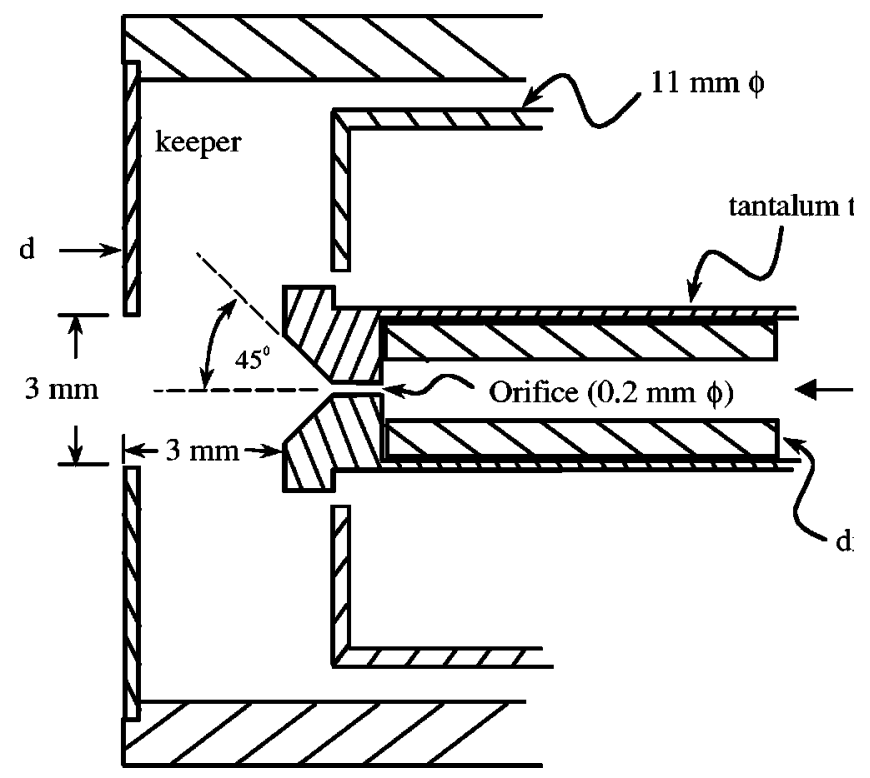

FIG. 2. Schematic cross section of the nozzle/keeper configuration.

with a downstream full-angle chamfer of $90^{\circ}$. A keeper electrode with $3 \mathrm{~mm}$ diameter aperture is positioned just downstream in an enclosed configuration. The hollow cathode had been operated previously. Changes in the operating point of the hollow cathode require a settling period, during which the device comes to a static condition. The duration of this period is typically $10-15 \mathrm{~min}$. The underlying cause of the settling behavior is believed to be a long time constant for returning to the set flow rate through the orifice. This phenomenon in itself provides some indication of the unusual nature of this type of hollow cathode.

The hollow cathode is installed in a $75-\mathrm{cm}$-diameter vacuum chamber, pumped by a $1000 \mathrm{l} / \mathrm{s}$ (on nitrogen) turbomolecular pump and a $1000 \mathrm{l} / \mathrm{s}$ (on xenon) cryopump. The base pressure with no xenon flow is about $2 \times 10^{-7}$ Torr. With a xenon flow rate of $0.105 \mathrm{mg} / \mathrm{s}$ through the hollow cathode, the background pressure indicated by an ion gauge positioned far from the cryopump is about $1.5 \times 10^{-5}$ Torr, after applying a standard sensitivity correction for xenon.

The cathode is installed on a rotation table in the vacuum chamber, with a QMS positioned behind a fixed beam skimmer, and an RPA also viewing the plume near the orifice (see Fig. 2). The range of rotary motion for the hollow cathode is $-50^{\circ}$ to $+90^{\circ}$ with respect to the axis of the QMS. The 5 $\mathrm{mm}$ aperture of the grounded skimmer is about $25 \mathrm{~mm}$ downstream from the keeper orifice. The entrance of the QMS is either 22 or $48 \mathrm{~cm}$ further downstream, and aligned with the hollow cathode orifice and beam skimmer.

The RPA consists of a Faraday cup with four closely spaced grids at the input. The entrance and third grid are grounded, and the fourth or innermost is biased negative to reject plasma electrons and to suppress the loss of secondary electrons. The second grid operates at the retarding potential, $V_{r}$. The acceptance area of the RPA is $1.0 \mathrm{~cm}^{2}$. The aperture is located $21 \mathrm{~cm}$ from the cathode orifice.

Two Langmuir probes constructed with $1 \mathrm{~mm}$ tungsten wire and shielded with ceramic insulator, are mounted verti- 
TABLE I. Hollow cathode conditions at various operating points.

\begin{tabular}{ccccc}
\hline \hline $\begin{array}{c}\text { Operating } \\
\text { point }\end{array}$ & $\begin{array}{c}\text { Keeper } \\
\text { current (A) }\end{array}$ & $\begin{array}{c}\text { Keeper } \\
\text { voltage }(\mathrm{V})\end{array}$ & $\begin{array}{c}\text { Flow } \\
\text { rate }(\mathrm{mg} / \mathrm{s})\end{array}$ & $\begin{array}{c}\text { Exit } \\
N_{e}\left(\mathrm{~m}^{-3}\right)\end{array}$ \\
\hline $\mathrm{A}$ & 1.12 & 26.0 & 0.10 & $3.4 \times 10^{20}$ \\
$\mathrm{~B}$ & 1.77 & 28.2 & 0.054 & $1.85 \times 10^{20}$ \\
$\mathrm{C}$ & 1.30 & 34.0 & 0.045 & $1.6 \times 10^{20}$ \\
\hline \hline
\end{tabular}

cally in the plume. The probes, designated L1 and L2 in Fig. 2 , are placed at $\sim 35$ and $110 \mathrm{~mm}$, respectively, from the keeper. In each case a $3 \mathrm{~mm}$ length of the tungsten wire is exposed to the plume.

\section{NUMERICAL MODEL}

\section{A. Hollow cathode plume model}

To understand the type of numerical approach required to accurately model the plasma plume from the hollow cathode, it is informative to consider the expected range of some of the basic physical properties of the flow. Using the geometric and physical information of the hollow cathode, at a typical operating point (three different operating points are listed in Table I), a 0-d hollow cathode device model of Domonkos ${ }^{11}$ is used to estimate the flow conditions at the exit of the hollow cathode, and some of these are included in Table I. For all points, the plasma density is of the order of $10^{20} \mathrm{~m}^{-3}$. The electron temperature is expected to be between 1 and $2 \mathrm{eV}$ for a xenon hollow cathode operated in plume mode (as is the case here), based on measurements reported by Domonkos ${ }^{11}$ in the orifice to keeper gap of a similar device. For operating point A, the electron-ion collision rate is about $5 \times 10^{9} \mathrm{~s}^{-1}$ giving an ion mean free path of about $3 \times 10^{-7} \mathrm{~m}$. This gives a Knudsen number based on the orifice exit radius of 0.0003 indicating thermal equilibrium conditions. Therefore it is reasonable to assume that the ion and electron temperatures are equal at the orifice exit. Then, using the mass flow rate, it is indicated that the plasma exiting the hollow cathode is very close to being fully ionized. These flow conditions indicate a very small Debye length so that charge neutrality can be safely assumed. While the mean free path is initially small, as the plasma plume expands the number density will decrease significantly thereby increasing the mean free path. These aspects of the plasma plume indicate that the electrons can be treated using a fluid approach, but the heavy particles require a kinetic description.

In this study, a hybrid particle-fluid model is therefore developed. The heavy particles (xenon ions and atoms) are treated using the particle in cell method $(\mathrm{PIC})^{12}$ to model their plasma dynamics, and the direct simulation Monte Carlo method (DSMC) ${ }^{13}$ is used to simulate their collision dynamics. The electrons are described by a detailed fluid model based on the conservation equations. These models are described in the following sections.

\section{B. Plasma dynamics}

Models of hollow cathode plumes have been developed by Parks et al. ${ }^{14}$ and by Williams and Wilbur. ${ }^{15}$ In Ref. 14, a fluid model of the electrons combined with an assumed profile for the ion density was used to model a mercury hollow cathode. By assuming anomalously low electrical conductivity (reduced by a factor of about 1000), good agreement was obtained for measurements of plasma potential and electron temperature.

The model under development in this work seeks to go beyond that described in Ref. 14 by explicitly modeling both the electrons and the heavy particles (ions and neutrals). Due to the low density nature of the hollow cathode plumes, a kinetic, particle approach is employed to simulate the xenon ions and neutral atoms. A detailed fluid model of the electrons is also employed.

The ions and neutrals are treated using a combination of the particle in cell method (PIC) ${ }^{12}$ for transporting the ions in electrostatic fields, and the direct simulation Monte Carlo method (DSMC) ${ }^{13}$ for performing collisions and transporting the neutral atoms. Momentum transfer and charge exchange collisions are the only collision mechanisms implemented at this stage.

The electron continuity equation is: ${ }^{16}$

$$
\frac{\partial}{\partial t}\left(N_{e}\right)+\nabla .\left(N_{e} \mathbf{v}_{\mathbf{e}}\right)=N_{e} N_{a} C_{i},
$$

where $N_{e}$ is the electron number density, $\mathbf{v}_{\mathbf{e}}$ is the electron velocity vector, $N_{a}$ is the atomic number density, and $C_{i}$ is the ionization rate coefficient. Assuming steady flow, this equation is transformed into a Poisson equation by introducing:

$$
N_{e} \mathbf{v}_{\mathbf{e}}=\nabla \psi
$$

such that

$$
\nabla^{2} \psi=N_{e} N_{a} C_{i}
$$

for which numerical solutions are obtained using the standard alternating direction implicit (ADI) method. The spatial distribution of the ion particles gives the electron number density, $N_{e}$, under the assumption of charge neutrality. This allows the electron velocity vector to be determined through solution of Eq. (3). The xenon ionization rate coefficient is expressed as a function of electron temperature using a simple relation proposed by Ahedo et al.: ${ }^{17}$

$$
C_{i}=\sigma_{i} c_{e}\left(1+\frac{T_{e} \varepsilon_{i}}{\left(T_{e}+\varepsilon_{e}\right)^{2}}\right) \exp \left(-\frac{\varepsilon_{i}}{T_{e}}\right),
$$

where $\sigma_{i}=5 \times 10^{-20} \mathrm{~m}^{2}$ is a reference cross section, $c_{e}$ is the mean thermal speed of electrons, $\varepsilon_{i}$ is the ionization energy of xenon $(12.7 \mathrm{eV})$, and the electron temperature $T_{e}$ is in electron volts. Use of this thermal ionization coefficient assumes that the electron stream velocity always lies below the threshold level for direct ionization of xenon (about 2 $\times 10^{6} \mathrm{~m} / \mathrm{s}$ ) which is true for all flow conditions considered.

The electron momentum equation is given by: ${ }^{16}$

$$
\frac{\partial}{\partial t}\left(m_{e} N_{e} \mathbf{v}_{\mathbf{e}}\right)+m_{e} N_{e}\left(\mathbf{v}_{\mathbf{e}} \cdot \nabla\right) \mathbf{v}_{\mathbf{e}}=-e N_{e} \mathbf{E}-\nabla p_{e}+\mathbf{R},
$$

where $m_{e}$ is the mass of an electron, $\mathbf{E}$ is the electric field, $p_{e}$ is the electron pressure, and $\mathbf{R}$ is the friction term. It is fur- 
ther assumed that the electrons behave as a perfect gas $\left(p_{e}\right.$ $\left.=N_{e} k T_{e}\right)$, and that the friction term is given by:

$$
\mathbf{R}=\frac{e N_{e} \mathbf{j}}{\sigma},
$$

where $\mathbf{j}$ is the current density, and $\sigma$ is the electrical conductivity.

Assuming a steady state, neglecting the inertial term on the left-hand side of Eq. (5), and introducing the plasma potential $-\nabla \phi=\mathbf{E}$, a generalized Ohm's law is obtained:

$$
\mathbf{j}=\sigma\left(-\nabla \phi+\frac{1}{e N_{e}} \nabla\left(N_{e} k T_{e}\right)\right) .
$$

For given $N_{e}, \mathbf{v}_{\mathbf{e}}$, and $T_{e}$, the charge continuity condition:

$$
\nabla . \mathbf{j}=0
$$

is then solved to obtain the plasma potential. This equation is written as a Laplace equation with weak source terms and is again solved using an ADI scheme.

The electron energy equation is given by: ${ }^{16}$

$$
\begin{gathered}
\frac{\partial}{\partial t}\left(\frac{3}{2} N_{e} k T_{e}\right)+\frac{3}{2} N_{e}\left(\mathbf{v}_{\mathbf{e}} \cdot \nabla\right) k T_{e}+p_{e} \nabla \cdot \mathbf{v}_{\mathbf{e}}=\nabla \cdot \kappa_{e} \nabla T_{e} \\
+\mathbf{j} . \mathbf{E}-3 \frac{m_{e}}{m_{i}} \nu_{e} N_{e} k\left(T_{e}-T_{H}\right)-N_{e} N_{a} C_{i} \varepsilon_{i},
\end{gathered}
$$

where $m_{i}$ is the ion mass, $\nu_{e}$ is the total electron collision frequency, $\kappa_{e}$ is the electron thermal conductivity, and $T_{H}$ is the heavy particle temperature. Again assuming a steady state, and dividing by the thermal conductivity:

$$
\begin{aligned}
\nabla^{2} T_{e}= & -\nabla \ln \left(\kappa_{e}\right) \cdot \nabla T_{e}+\frac{1}{\kappa_{e}}\left(-\mathbf{j} . \mathbf{E}+\frac{3}{2} N_{e}\left(\mathbf{v}_{\mathbf{e}} \cdot \nabla\right) k T_{e}\right. \\
& \left.+p_{e} \nabla \cdot \mathbf{v}_{\mathbf{e}}+3 \frac{m_{e}}{m_{i}} \nu_{e} N_{e} k\left(T_{e}-T_{H}\right)+N_{e} N_{a} C_{i} \varepsilon_{i}\right)
\end{aligned}
$$

where $\mathbf{j}$ is obtained from Eq. (7) after the plasma potential is calculated. Equation (10) is again a Laplace equation with weak source terms that is solved using the ADI approach. Effects of ionization are included in the simulation to capture any regions of significant xenon ion production and it is therefore appropriate to include the associated effects in the electron energy equation. However, the computations subsequently showed no significant effect of ionization on the electron energy and this is also found to be true for excitation loss terms. The latter are therefore omitted from the modeling.

Finally, the electron transport coefficients are evaluated using the basic definitions from: ${ }^{16}$

$$
\begin{gathered}
\sigma=\frac{e^{2} N_{e}}{m_{e} \nu_{e}}, \\
\kappa_{e}=\frac{2.4}{1+\frac{\nu_{e i}}{\sqrt{2} \nu_{e}}} \frac{k^{2} N_{e} T_{e}}{m_{e} \nu_{e}},
\end{gathered}
$$

where $\nu_{e}=\nu_{e i}+\nu_{e n}, \nu_{e i}$ is the ion-electron collision frequency, $\nu_{e n}$ is the neutral-electron collision frequency, and these frequencies are evaluated for the xenon system using cross sections provided in Ref. 16. Note that, for each time step, the numerical scheme iterates several times through the solution of Eqs. (8) and (9) due to the coupling between $\phi$ and $T_{e}$.

\section{Collision dynamics}

The DSMC method uses particles to simulate collision effects in rarefied gas flows by collecting groups of particles into cells which have sizes of the order of a mean free path. Pairs of these particles are then selected at random and a collision probability is evaluated that is proportional to the product of the relative velocity and collision cross section for each pair. The probability is compared with a random number to determine if that collision occurs. If so, some form of collision dynamics is performed to alter the properties of the colliding particles.

There are two basic classes of collisions that are important in the hollow cathode plumes: (1) elastic (momentum exchange); and (2) charge exchange. Elastic collisions involve only exchange of momentum between the participating particles. For the systems of interest here, this may involve atom-atom or atom-ion collisions. For atom-atom collisions, the variable hard sphere $(\mathrm{VHS})^{13}$ collision model is employed. For xenon, the collision cross section is:

$$
\sigma_{E L}(\mathrm{Xe}, \mathrm{Xe})=\frac{2.12 \times 10^{-18}}{g^{2 \omega}} \mathrm{m}^{2},
$$

where $g$ is the relative velocity, and $\omega=0.12$ is related to the viscosity temperature exponent for xenon. For atom-ion elastic interactions, the following cross section of Dalgarno et al. ${ }^{18}$ is employed:

$$
\sigma_{E L}\left(\mathrm{Xe}_{\mathrm{e}, \mathrm{Xe}^{+}}\right)=\frac{6.42 \times 10^{-16}}{g} \mathrm{~m}^{2} .
$$

The model of Ref. 18 predicts that the elastic cross section for interaction between an atom and a doubly charged ion is twice that for an atom and a singly charged ion. It should be noted that the model of Ref. 18 employs a polarization potential and therefore is only valid for low energy (a few eV) collisions. In all elastic interactions, the collision dynamics is modeled using isotropic scattering together with conservation of linear momentum and energy to determine the postcollision velocities of the colliding particles. ${ }^{13}$

Charge exchange concerns the transfer of one or more electrons between an atom and an ion. For singly charged ions, the following cross section measured by Pullins et al. ${ }^{19}$ and Miller et al. ${ }^{20}$ is used:

$$
\begin{aligned}
\sigma_{C E X}\left(\mathrm{Xe}, \mathrm{Xe}^{+}\right)= & {\left[-23.30 \log _{10}(g)+142.21\right] } \\
& \times 0.8423 \times 10^{-20} \mathrm{~m}^{2} .
\end{aligned}
$$

Also reported in Refs. 19 and 20 are charge exchange cross sections for the interaction where a doubly charged ion captures two electrons from an atom. These cross sections are less than a factor of two lower than the values for the singly charged ions at corresponding energies. In the present model, 
it is assumed that there is no transfer of momentum accompanying the transfer of the electron(s). This assumption is based on the premise that charge exchange interactions are primarily at long range.

\section{Boundary conditions}

For the computations of the hollow cathode plumes, boundary conditions must be specified at several locations: (1) at the cathode orifice exit; (2) along the outer edges of the computational domain; and (3) along all solid surfaces in the computational domain.

Several macroscopic properties of the plasma exiting the cathode orifice are required for the computations. Specifically, the plasma potential, the electron temperature, and for each of the particle species we require the number density, velocity, and temperature. In the real device, these properties vary radially across the cathode exit plane. As described above, the approach to determining these properties involves a mixture of analysis and estimation. The plasma number density is obtained using the device model of Domonkos. ${ }^{11}$ The electron temperature is set to $1.5 \mathrm{eV}$ as a representative value based on the measurements reported in Ref. 11. Using the specified mass flow rate and assuming the ion temperature is equal to the electron temperature, the plasma is found to be nearly fully ionized. The fraction of $\mathrm{Xe}^{2+}$ is assumed to be $10 \%$.

Both fluid and particle boundary conditions are required at the outer edges of the computational domain. The usual field conditions employed simply set the electric fields normal to the boundary edges equal to zero. Similarly the gradients in electron temperature normal to the surfaces of the outer boundaries are set to zero. For plume expansion into vacuum, the particle boundary condition is to simply remove from the computation any particle crossing the domain edge.

The solid wall surfaces of the hollow cathode and keeper are included in the computation. The potentials are set to specified constants (the keeper voltage for the keeper walls, zero for all other walls). Zero gradient in electron temperature is again employed on all walls. Any ions colliding with the walls are neutralized. Both atoms and neutralized ions are scattered back into the flow field from the surface of the wall assuming diffuse reflection at a temperature of $1000^{\circ} \mathrm{C}$.

\section{RESULTS}

The hollow cathode plume model described above is applied to three different operating points as listed in Table I. In the following, general flow field results of the model are first presented. Next, the physical accuracy of the model is assessed by making direct comparison with measured data. In these comparisons, a number of basic assumptions in the model are varied to understand the sensitivity of the computed results.

In all cases, the computational domain extends to $0.30 \mathrm{~m}$ along the axis from the orifice and $0.25 \mathrm{~m}$ radially from the centerline. A portion of the grid is shown in Fig. 3 and employs 110 by 90 nonuniform, rectangular cells. A time-step of $5 \times 10^{-9} \mathrm{~s}$ is employed, which is smaller than the smallest inverse plasma frequency. The simulation typically reaches a steady state after about 25000 iterations and final results are

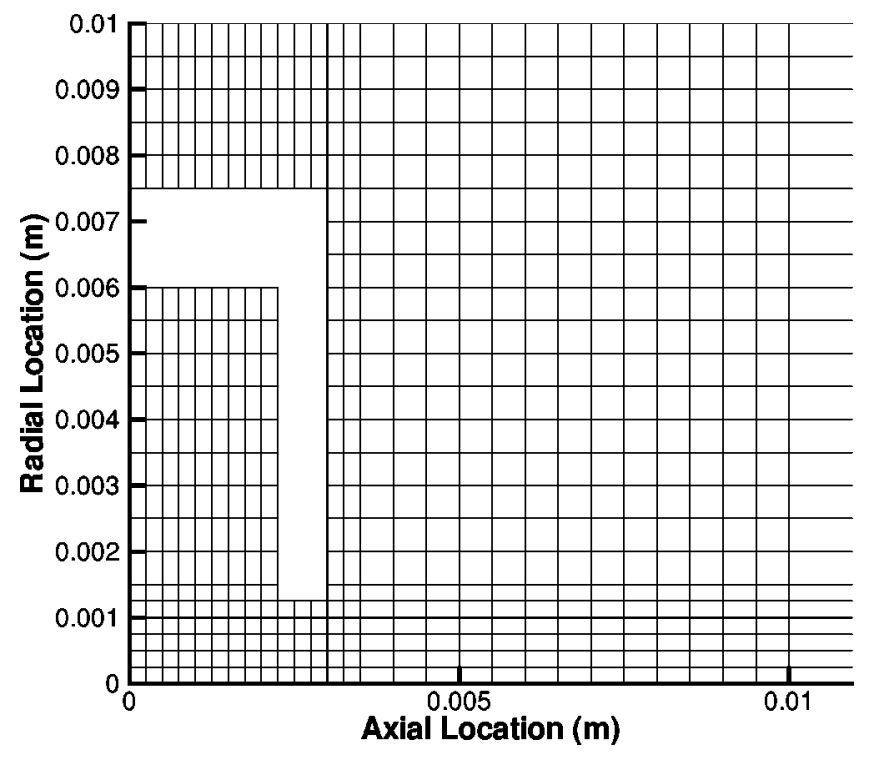

FIG. 3. Portion of the computational grid showing the structure of the keeper.

obtained by averaging over a further 20000 iterations. About 1.5 million particles are employed in each simulation.

\section{A. Results for operating point $A$}

Typical flow field properties are first considered for operating point $\mathrm{A}$ (see Table I) for conditions assumed at the cathode orifice exit of $T_{e}=T_{i}=1.5 \mathrm{eV}$, and $\phi=$ keeper voltage. Figures 4, 5, 6, and 7 show the electron density $\left(\mathrm{m}^{-3}\right)$, the plasma potential $(\mathrm{V})$, the electron velocity component in the axial direction $(\mathrm{m} / \mathrm{s})$, and the electron velocity component in the radial direction $(\mathrm{m} / \mathrm{s})$. Note that the computed potentials are always presented with respect to cathode (ground) potential. There is not a significant variation in the electron temperature. These results show that the plasma ex-

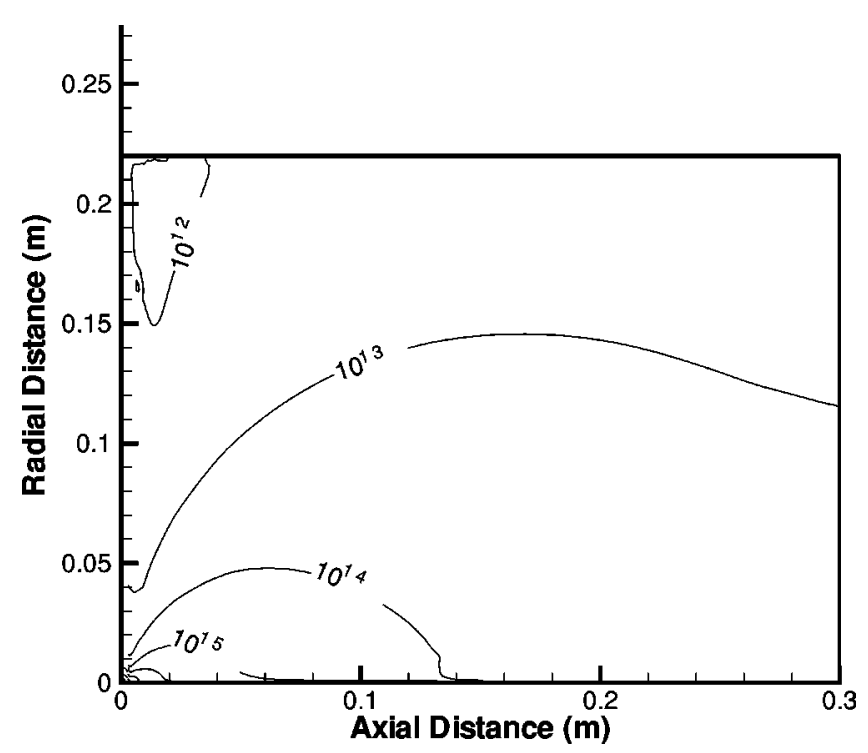

FIG. 4. Contours of electron density for operating point A. 


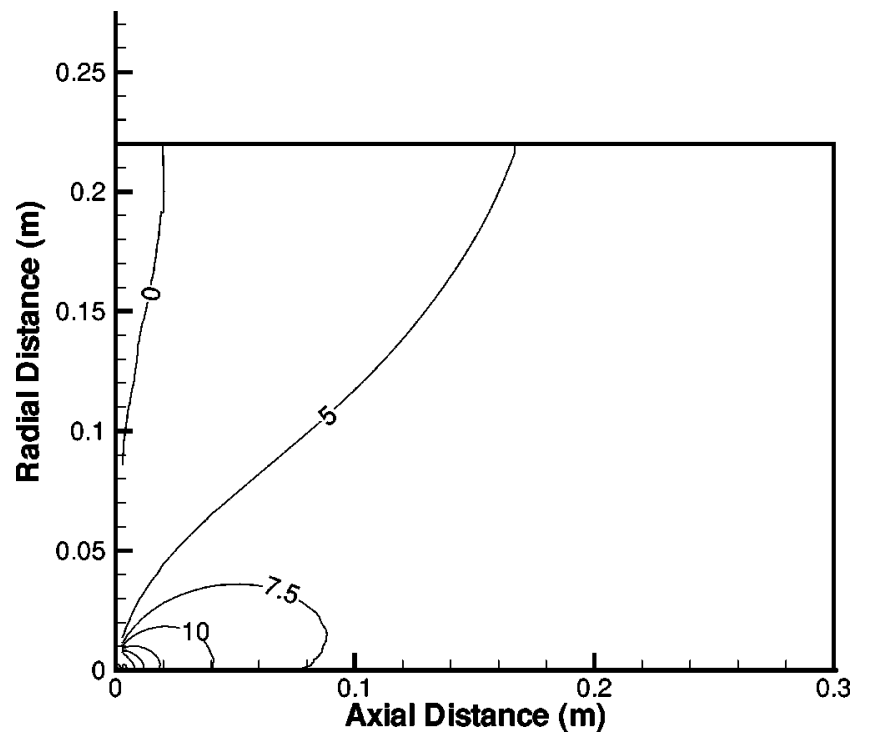

FIG. 5. Contours of plasma potential for operating point A.

pands through the orifice of the keeper to rather low plasma densities. There is a thin jet of relatively high density plasma right on the axis. This is formed by the heavy ions becoming highly directional after being forced through the opening in the keeper plate. The potential contours indicate that the resulting electric fields radial to the axis are not sufficiently strong to subsequently diffuse these ions away from the axis. The axial velocity contours show that the high keeper voltage decelerates the electrons and turns them around at a distance of about $20 \mathrm{~cm}$ from the keeper for this particular case.

Next, the sensitivity of the computational results to several key assumptions is investigated. First, we consider the effect of varying the initial value of electron (and ion) temperature assumed at the orifice exit. Figure 8 shows the effect of varying the initial electron temperature on profiles of electron temperature and plasma potential along the flow axis. The profiles of electron temperature simply scale up and

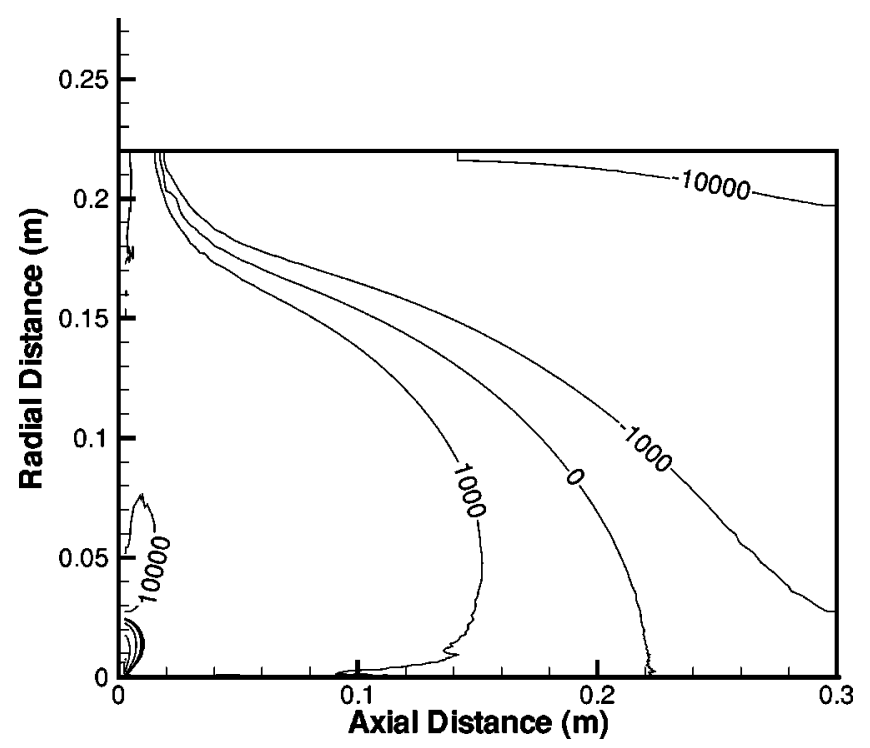

FIG. 6. Contours of electron axial velocity component for operating point A.

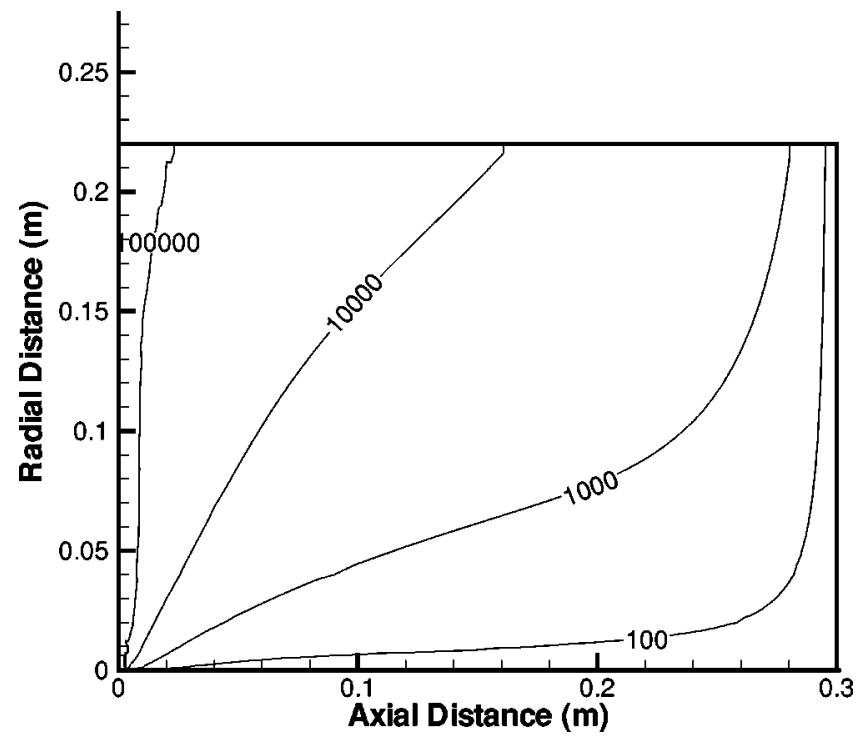

FIG. 7. Contours of electron radial velocity component for operating point A.

down as the initial value of $T_{e}$ is increased and decreased from the default value of $1.5 \mathrm{eV}$. A more dramatic effect is observed for the plasma potential in which both the maximum potential is larger and the minimum potential smaller in the case of increased $T_{e}$. This behavior is expected to give larger ion energies for this condition. In Fig. 9, the effect on profiles along the axis of electron density and the axial component of the electron velocity is investigated. Note that each of these properties is relatively insensitive to the initial value of $T_{e}$. This indicates that current is insensitive to $T_{e}$.

Now, let us consider the effects of varying $T_{e}$ on properties for which experimental measurements exist. In Fig. 10, ion energy distribution functions (IEDFs) obtained on axis at $21 \mathrm{~cm}$ from the keeper exit are shown. The computed distributions are obtained in the simulation by binning ion energies of individual particles crossing the location in the flow field where the RPA is placed. Because the RPA entrance is

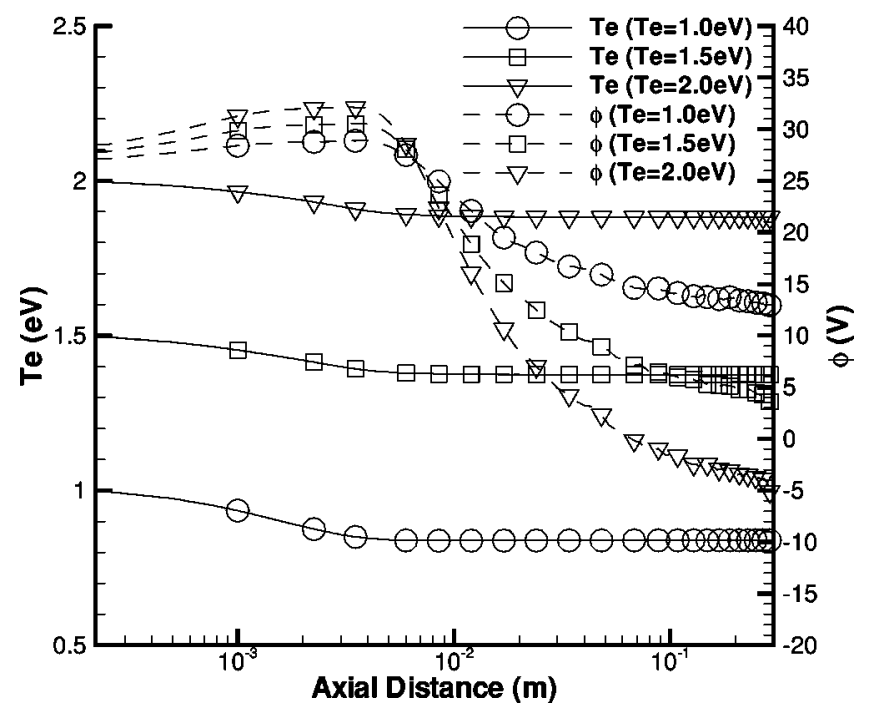

FIG. 8. Profiles of electron temperature and plasma potential along the axis for operating point A: Effect of varying initial electron temperature. 


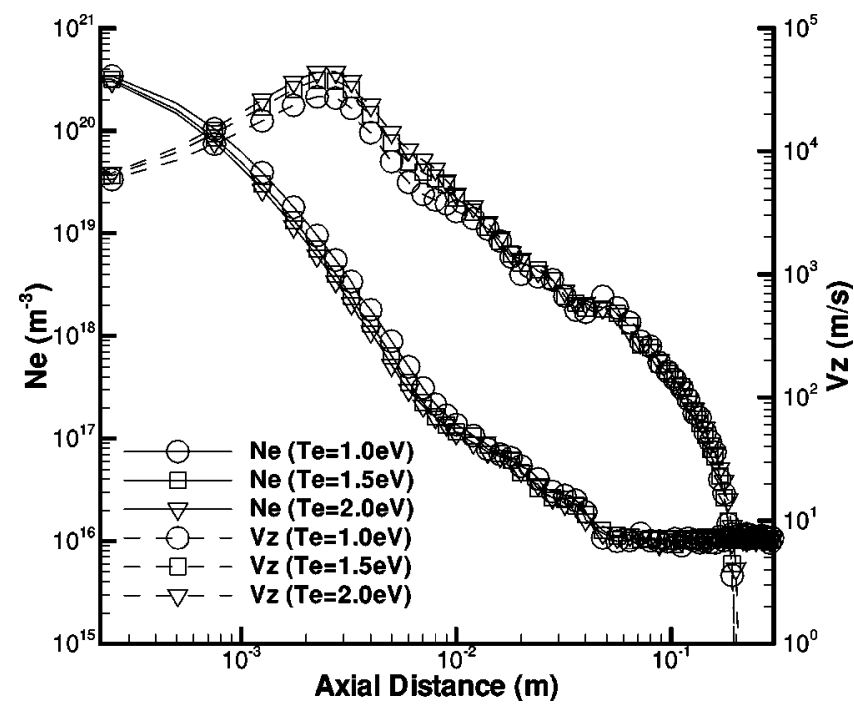

FIG. 9. Profiles of electron density and electron axial velocity along the axis for operating point A: Effect of varying initial electron temperature.

grounded in the experiment, after the simulation is completed, the computed IEDF is shifted by the difference between the plasma potential in the simulation at the location of the RPA and ground $(0 \mathrm{~V})$. The shifts in potential vary widely for the simulations performed and are listed in Table II for all conditions considered in this study. As expected, the computed IEDFs are sensitive to $T_{e}$ with slightly higher peak ion energies obtained at higher values of $T_{e}$. Note also that the width of the computed distributions increases with increasing $T_{e}$. All three simulations provide a good prediction of the peak of the IEDF and underpredict the measured width. Note that the high energy tails of the computed distributions are not unexpected. For example, the highest ion energy for the $T_{e}=2.0 \mathrm{eV}$ case is about $60 \mathrm{~V}$ when the offset is accounted for. Figure 8 shows that the overall change in potential along the centerline from peak to trough is about 38

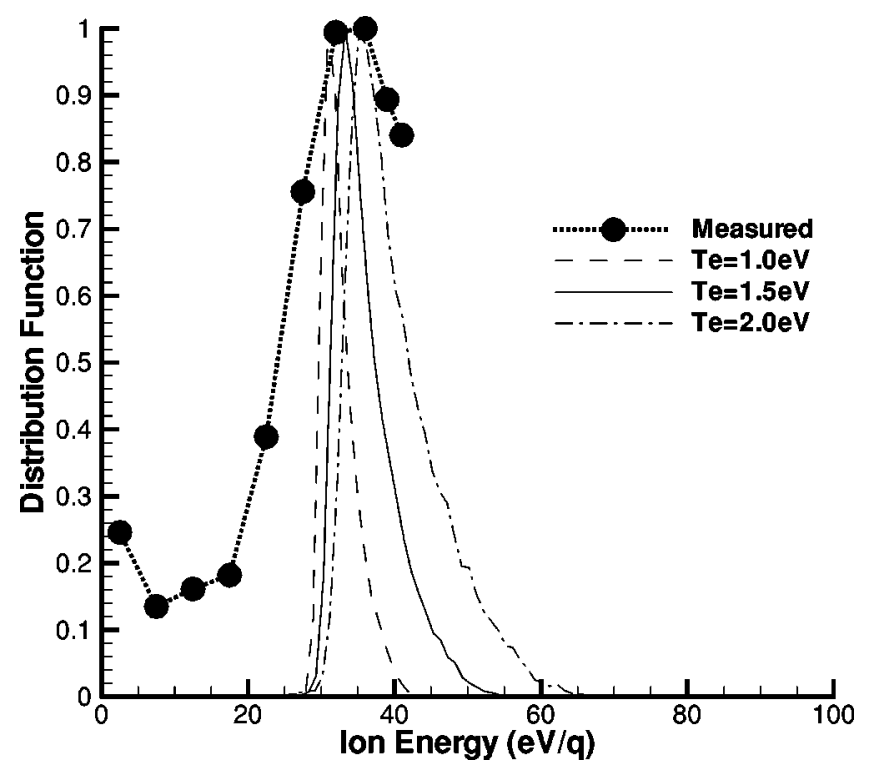

FIG. 10. Ion energy distribution at $21 \mathrm{~cm}$ from the hollow cathode exit for operating point A: Effect of varying initial electron temperature.
TABLE II. Shift in computed IEDFs to correct for grounded RPA for all simulations.

\begin{tabular}{ccccc}
\hline \hline $\begin{array}{c}\text { Operating } \\
\text { point }\end{array}$ & $\begin{array}{c}T_{e} \\
(\mathrm{eV})\end{array}$ & $\begin{array}{c}\Delta \phi \\
(\mathrm{V})\end{array}$ & $\begin{array}{c}\nu_{e i} \\
\text { factor }\end{array}$ & $\begin{array}{c}\text { Potential } \\
\text { shift }(\mathrm{V})\end{array}$ \\
\hline $\mathrm{A}$ & 1.5 & 0 & 1 & +4.8 \\
$\mathrm{~A}$ & 1.0 & 0 & 1 & +13.4 \\
$\mathrm{~A}$ & 2.0 & 0 & 1 & -3.3 \\
$\mathrm{~A}$ & 1.5 & -2 & 1 & +2.24 \\
$\mathrm{~A}$ & 1.5 & -6 & 1 & -4.39 \\
$\mathrm{~A}$ & 1.5 & 0 & 0.1 & +3.82 \\
$\mathrm{~A}$ & 1.5 & 0 & 10 & +14.9 \\
$\mathrm{~B}$ & 1.75 & -2 & 1 & +1.05 \\
$\mathrm{C}$ & 1.5 & 0 & 1 & +13.64 \\
$\mathrm{C}$ & 2.5 & 0 & 1 & -26.3 \\
$\mathrm{C}$ & 4.0 & 0 & 1 & -5.62 \\
$\mathrm{C}$ & 2.5 & -2 & 1 & -13.4 \\
$\mathrm{C}$ & 2.5 & -6 & 1 & -3.49 \\
$\mathrm{C}$ & 2.5 & 0 & 0.1 & +2.15 \\
$\mathrm{C}$ & 2.5 & 0 & 10 & +15.4 \\
$\mathrm{C}$ & 2.5 & 0 & 30 & -1.9 \\
$\mathrm{C}$ & 4.0 & 0 & 70 &
\end{tabular}

$\mathrm{V}$ for this case. An ion introduced into the simulation at the cathode exit following an equilibrium energy distribution at $2.0 \mathrm{eV}$ has a probability of about $10^{-4}$ of having the initial energy of $22 \mathrm{~V}$ needed to reach $60 \mathrm{~V}$ at the location of the RPA.

In Fig. 11, angular profiles of ion current obtained at a distance of $21 \mathrm{~cm}$ from the keeper are shown. The computational results are relatively insensitive to the value of $T_{e}$ assumed, and all lie above the measured data by about a factor of two. With the observation that current is insensitive to $T_{e}$, Eq. (7) indicates that changes in $T_{e}$ must be balanced by changes in $\nabla \phi$.

Next, the effect of varying the value of plasma potential at the orifice exit on the simulation results is investigated. The parameter varied is $\Delta \phi$ which is the difference between the value of $\phi$ assumed at the exit, and the keeper potential. Measurements in the orifice-keeper gap of similar hollow

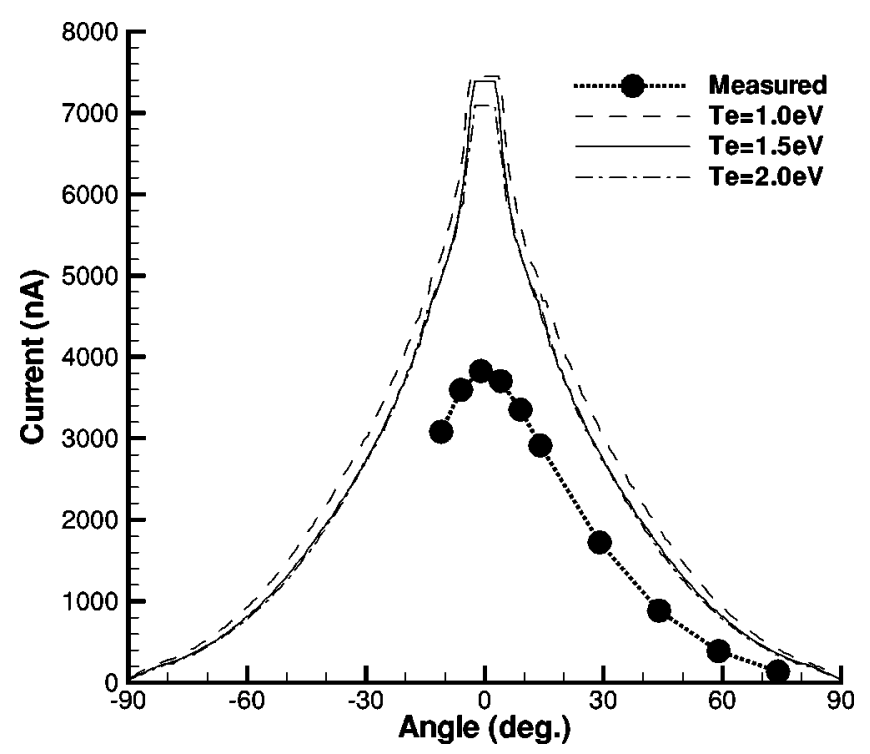

FIG. 11. Angular profile of ion current at $21 \mathrm{~cm}$ from the hollow cathode exit for operating point A: Effect of varying initial electron temperature. 


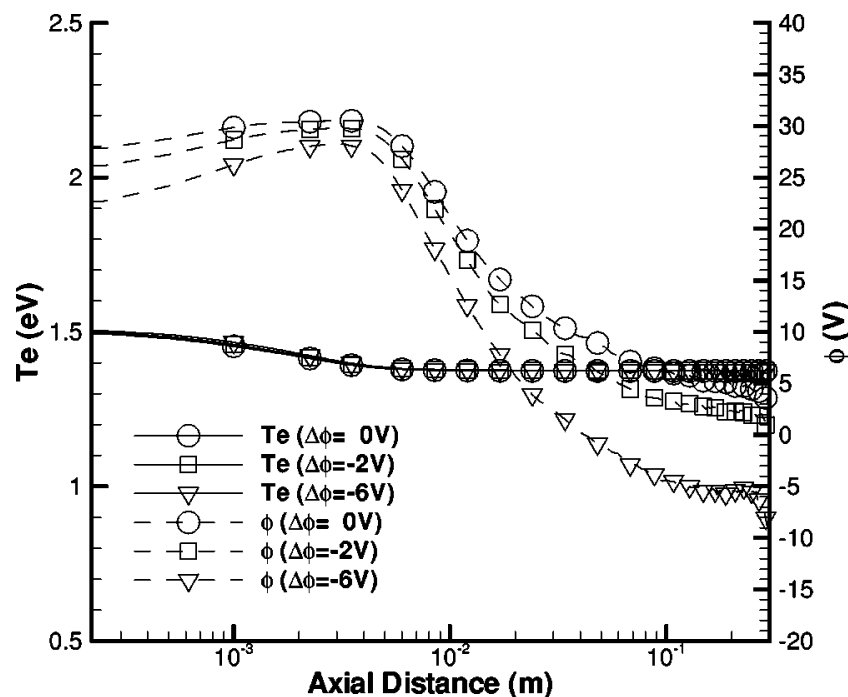

FIG. 12. Profiles of electron temperature and plasma potential along the axis for operating point A: Effect of varying initial plasma potential.

cathodes by Domonkos ${ }^{11}$ indicate that $\Delta \phi$ lies in the range of 0 to $-2 \mathrm{~V}$. Figure 12 shows that electron temperature is unaffected by the value of $\Delta \phi$. The profiles of plasma potential indicate that a lower value of $\Delta \phi$ produces a larger overall change in the potential that is expected to generate higher energy ions. In Fig. 13, it is shown that variation of $\Delta \phi$ affects both the electron number density and velocity profiles along the axis. Specifically, lower values of $\Delta \phi$ lead to greater initial acceleration of the electrons to the keeper accompanied by lower electron density. It is anticipated that this behavior will lead to lower ion current at the lower values of $\Delta \phi$. Comparing to the measured data, Fig. 14 indicates a corresponding shift in the most probable ion energy as $\Delta \phi$ is decreased, along with a significant decrease in ion current as shown in Fig. 15.

Finally, the effect of varying the values of the thermal and electron conductivities in the simulation is investigated.

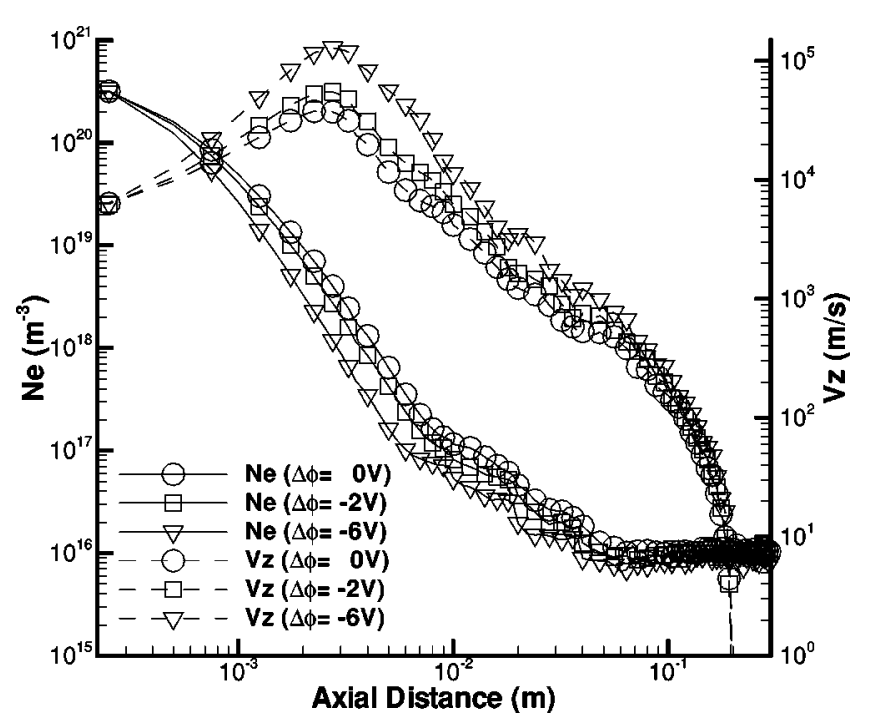

FIG. 13. Profiles of electron density and electron axial velocity along the axis for operating point A: Effect of varying initial plasma potential.

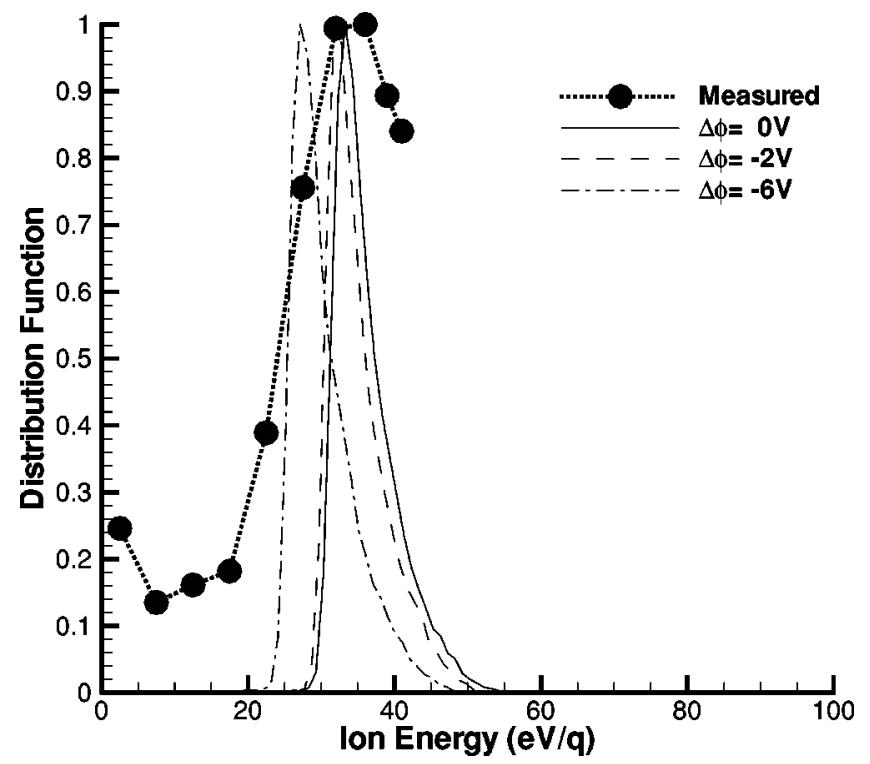

FIG. 14. Ion energy distribution at $21 \mathrm{~cm}$ from the hollow cathode exit for operating point A: Effect of varying initial plasma potential.

This study is motivated by the suggestion in Ref. 14 that electron-ion instabilities in hollow cathode plume flows may effectively increase the electron-ion collision rate, $\nu_{e i}$, to values close to the plasma frequency. In the present work, the sensitivity of the solutions to the transport coefficients is investigated by increasing and decreasing the values of $\nu_{e i}$ in the simulation by a factor of 10 in each case. From Eqs. (11), an increase in $\nu_{e i}$ leads to a decrease in both the thermal and electrical conductivities. Figure 16 indicates that the profiles along the axis of the electron temperature are very sensitive to the transport coefficients. In particular, increased values of $\nu_{e i}$ lead to a significant decrease in the electron temperature. Careful analysis of the results indicates that the rapid decrease in electron temperature is caused by amplification of the $p_{e} \nabla \cdot \mathbf{v}_{\mathbf{e}}$ term on the right-hand side of Eq. (10). In the

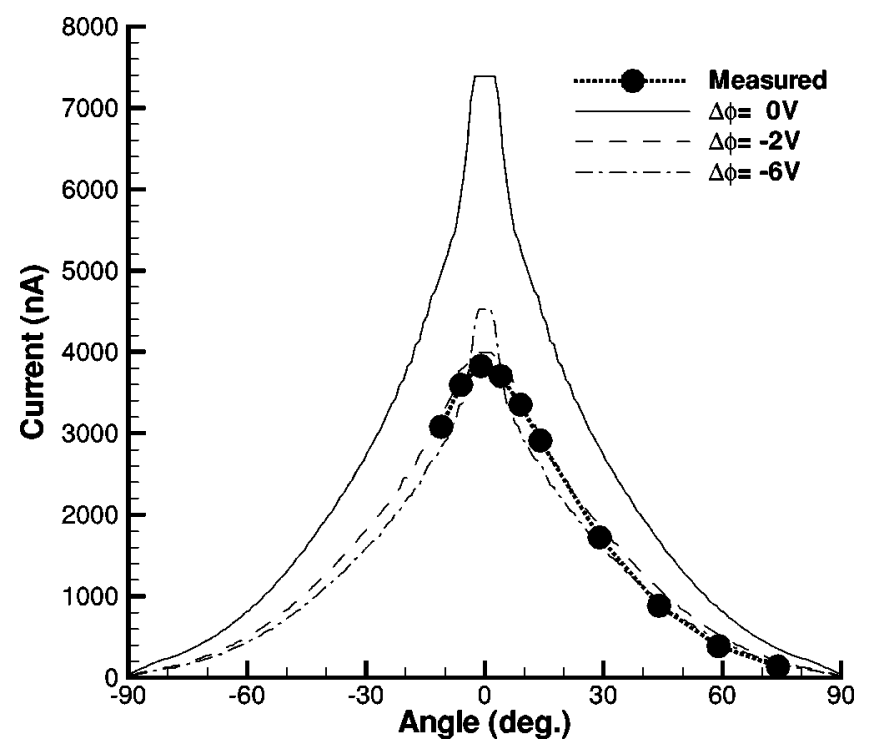

FIG. 15. Angular profile of ion current at $21 \mathrm{~cm}$ from the hollow cathode exit for operating point A: Effect of varying initial plasma potential. 


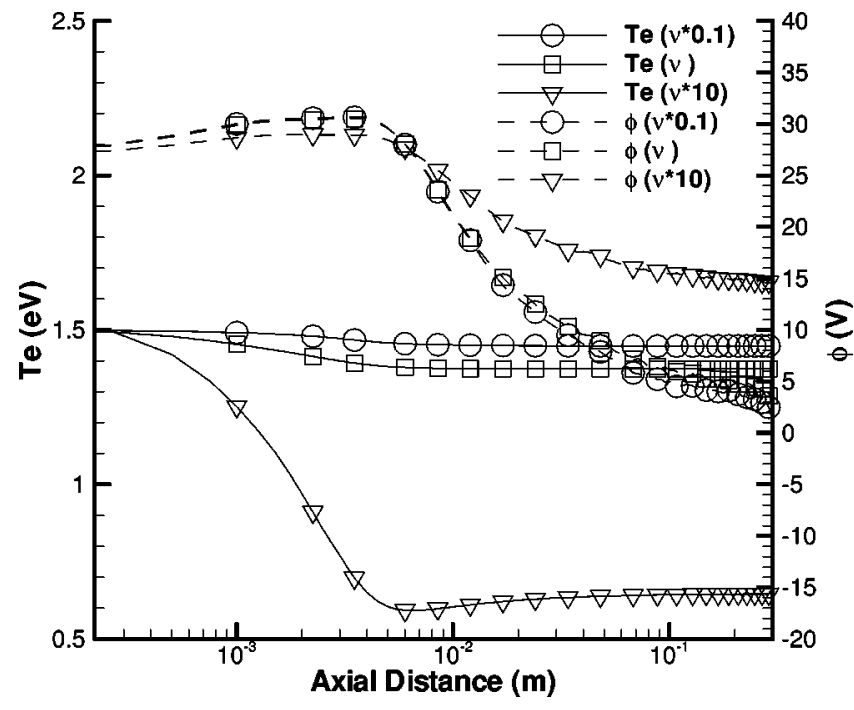

FIG. 16. Profiles of electron temperature and plasma potential along the axis for operating point A: Effect of varying transport coefficients.

region between the cathode orifice exit and the keeper, the electrons are accelerated and therefore expand leading to cooling of their temperature. This cooling is moderated by the ohmic heating $\mathbf{j} . \mathbf{E}$ term, but the overall effect is cooling. The magnitude of the cooling is increased when $\nu_{e i}$ is increased because in turn this leads to decreased values of the thermal conductivity, $\kappa_{e}$. The pressure expansion term was not included in the energy equation used in the work of Park et al. ${ }^{14}$ which explains why they observed increases in $T_{e}$. Another effect found in our results for increased $\nu_{e i}$, is that the gradient in the plasma potential is greatly decreased. Thus, lower energy ions are expected for this case. The profiles in Fig. 17 show that the electron velocity is generally lower and the electron density generally higher for the case of increased $\nu_{e i}$. Figure 18 indicates very little effect on the IEDF of the values of $\nu_{e i}$. By comparison, Fig. 19 shows that the ion current increases as $\nu_{e i}$ is increased.

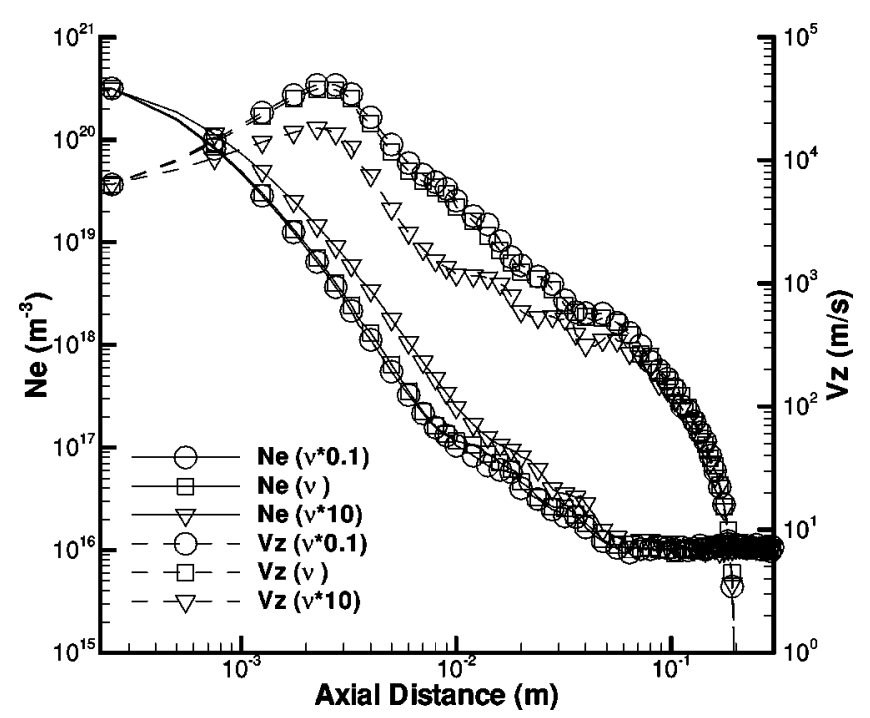

FIG. 17. Profiles of electron density and electron axial velocity along the axis for operating point A: Effect of varying transport coefficients.

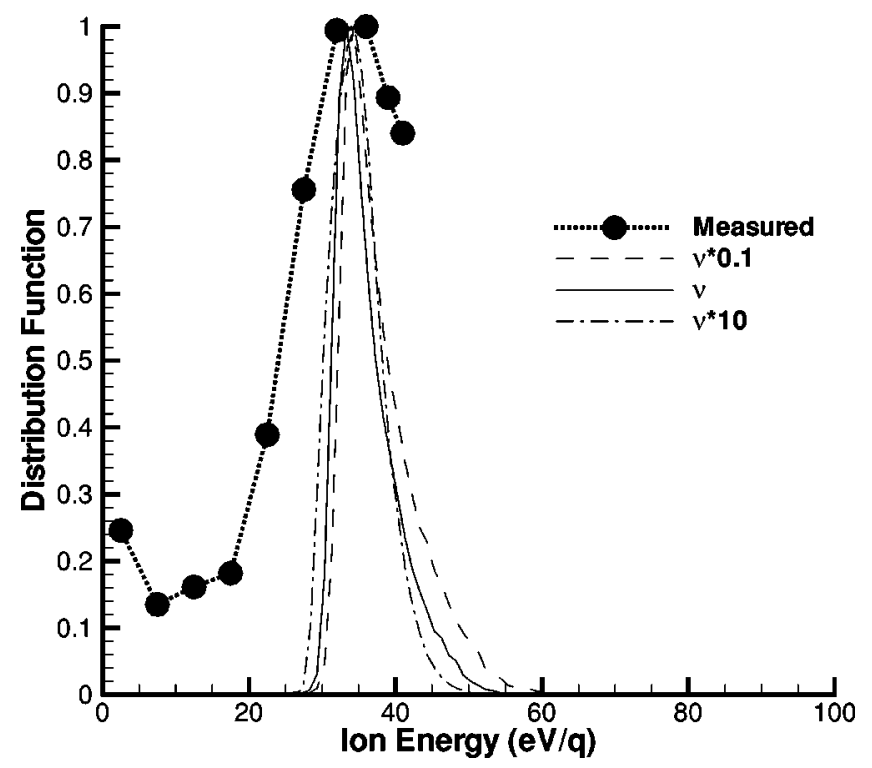

FIG. 18. Ion energy distribution at $21 \mathrm{~cm}$ from the hollow cathode exit for operating point A: Effect of varying transport coefficients.

Overall, for operating point A, the best agreement between the model and the measurements is obtained for both ion energy distribution and angular profile of ion current for values of $T_{e}=2.0 \mathrm{eV}, \Delta \phi=-2 \mathrm{~V}$, and the standard values for the transport coefficients. These are all reasonable, indicating satisfactory performance of the model.

\section{B. Results for operating point B}

As further evidence of the reasonable behavior of the model, it should be noted that a Langmuir probe measurement of centerline plasma potential and electron temperature of the present hollow cathode was performed at operating point B (similar to operating point A), at a distance of $35 \mathrm{~mm}$ downstream from the keeper. Comparison of these measured data with on-axis simulation results is given in Fig. 20. The

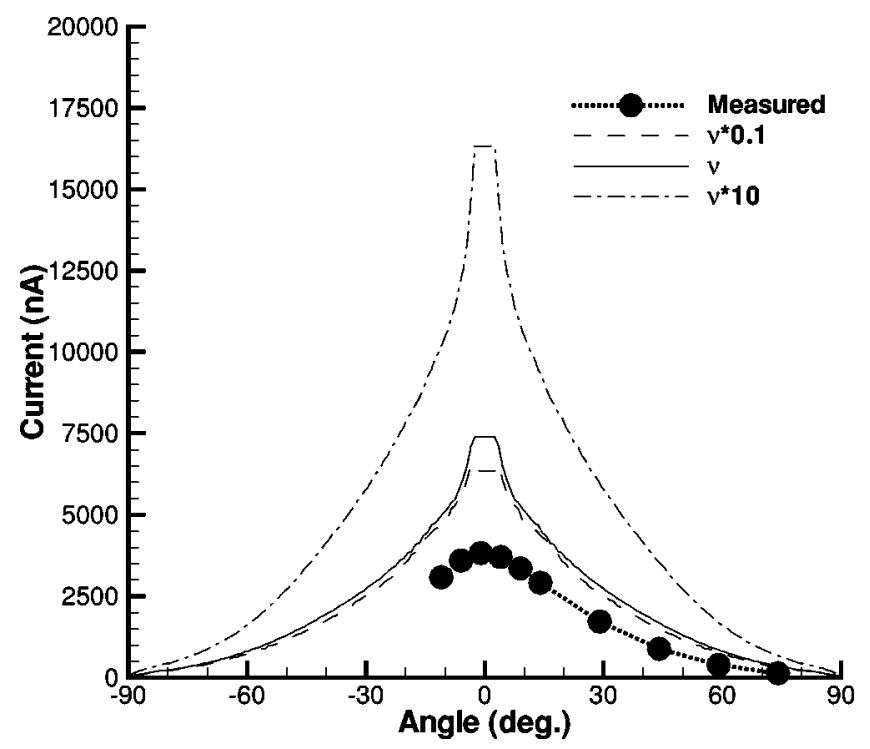

FIG. 19. Angular profile of ion current at $21 \mathrm{~cm}$ from the hollow cathode exit for operating point A: Effect of varying transport coefficients. 


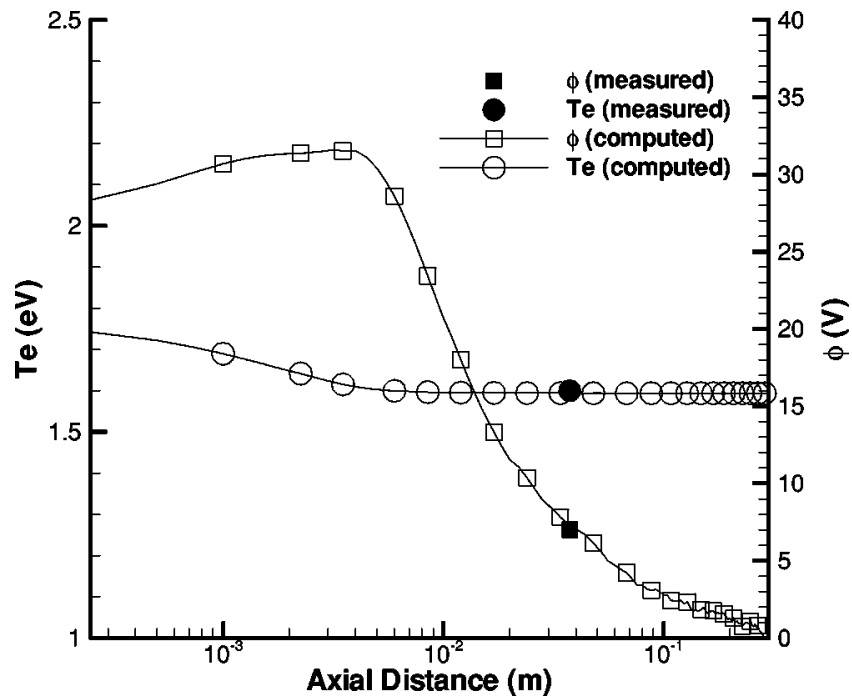

FIG. 20. Profiles of electron temperature and plasma potential along the axis for operating point $\mathrm{B}$ : Comparison with measured data.

simulation employs values of $T_{e}=T_{i}=1.75 \mathrm{eV}$, and $\Delta \phi=$ $-2 \mathrm{~V}$ at the orifice exit, and the standard transport coefficients. The agreement obtained with the data is excellent. In addition, comparison between this simulation and the measured angular profile of ion current is provided in Fig. 21, and again good agreement is obtained. In terms of agreement between simulation and experiment, operating points $\mathrm{A}$ and $\mathrm{B}$ are very similar. Note that Langmuir probe data are only available for operating point $\mathrm{B}$, and that no ion energy distribution data were taken at this condition.

\section{Operating point $\mathrm{C}$}

Operating point $\mathrm{C}$ has the highest keeper voltage and lowest mass flow rate of the conditions considered. Electron temperature typically increases in drift tubes according to the reduced field, $E / n$, with approximately linear dependence. ${ }^{21}$

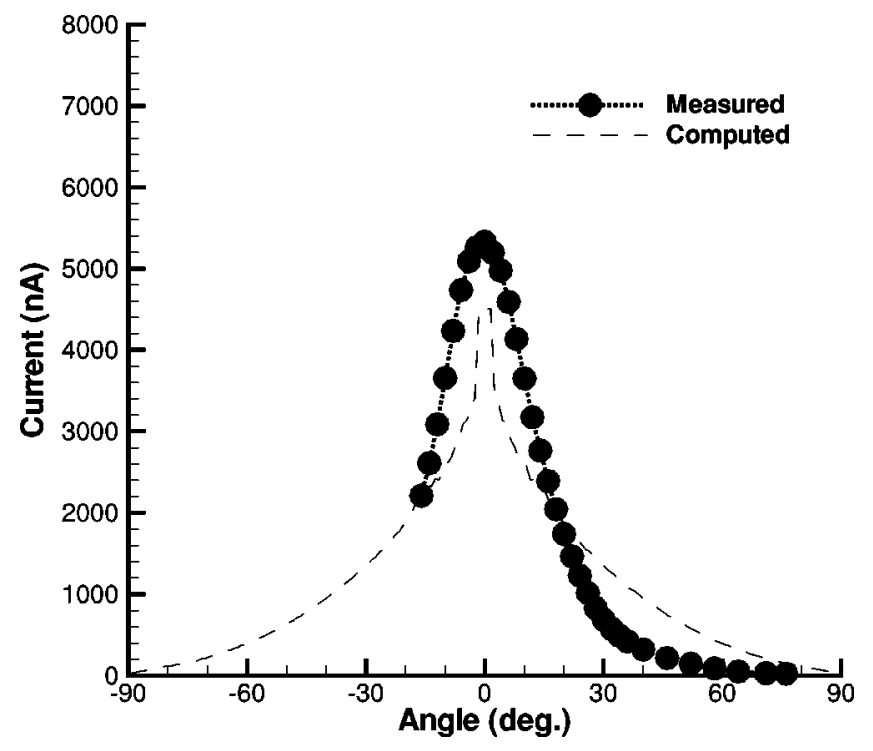

FIG. 21. Angular profile of ion current at $21 \mathrm{~cm}$ from the hollow cathode exit for operating point $\mathrm{B}$.

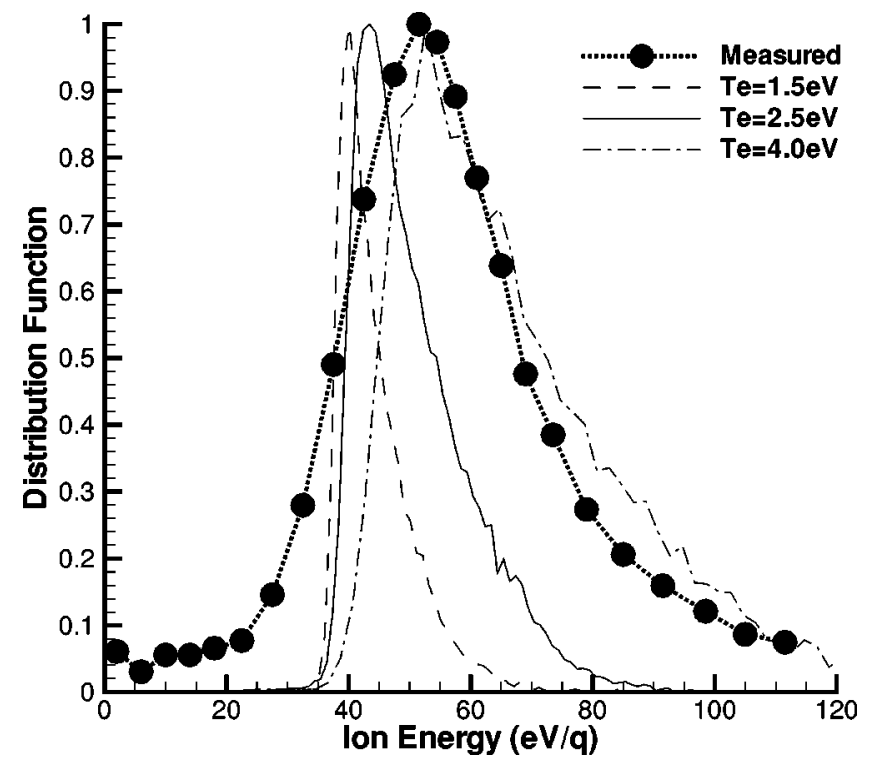

FIG. 22. Ion energy distribution at $21 \mathrm{~cm}$ from the hollow cathode exit for operating point $\mathrm{C}$ : Effect of varying initial electron temperature.

Of the operating points explored, this ratio is highest for operating point $\mathrm{C}$ and is lowest for operating point $\mathrm{A}$. Therefore it is reasonable to expect that electron temperature for operating point $\mathrm{C}$ will be larger than that for points $\mathrm{A}$ and $\mathrm{B}$. In Fig. 22, measured and computed ion energy distribution functions are compared. Here, the effect of varying electron temperature is studied ( $\Delta \phi=0 \mathrm{~V}$ and standard transport coefficients are used for these cases). The trends are the same as those for operating point $\mathrm{A}$, and, as suggested by our discussion above, for this case it requires an elevated initial electron and ion temperature $(4.0 \mathrm{eV})$ to obtain agreement with the measured data. However, Fig. 23 shows the data comparisons for ion current under the same conditions, and

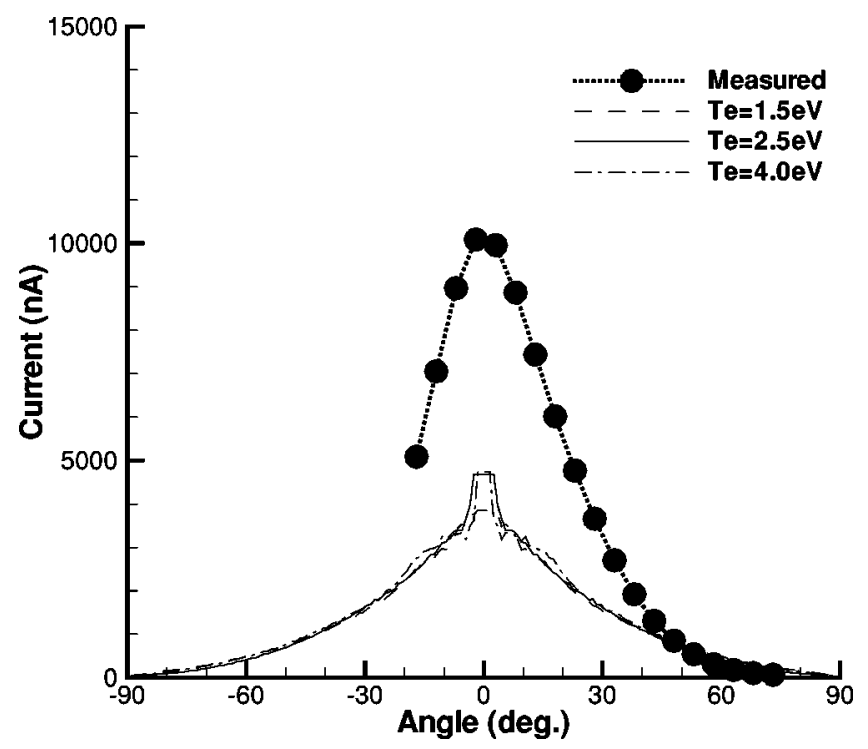

FIG. 23. Angular profile of ion current at $21 \mathrm{~cm}$ from the hollow cathode exit for operating point $\mathrm{C}$ : Effect of varying inital electron temperature. 


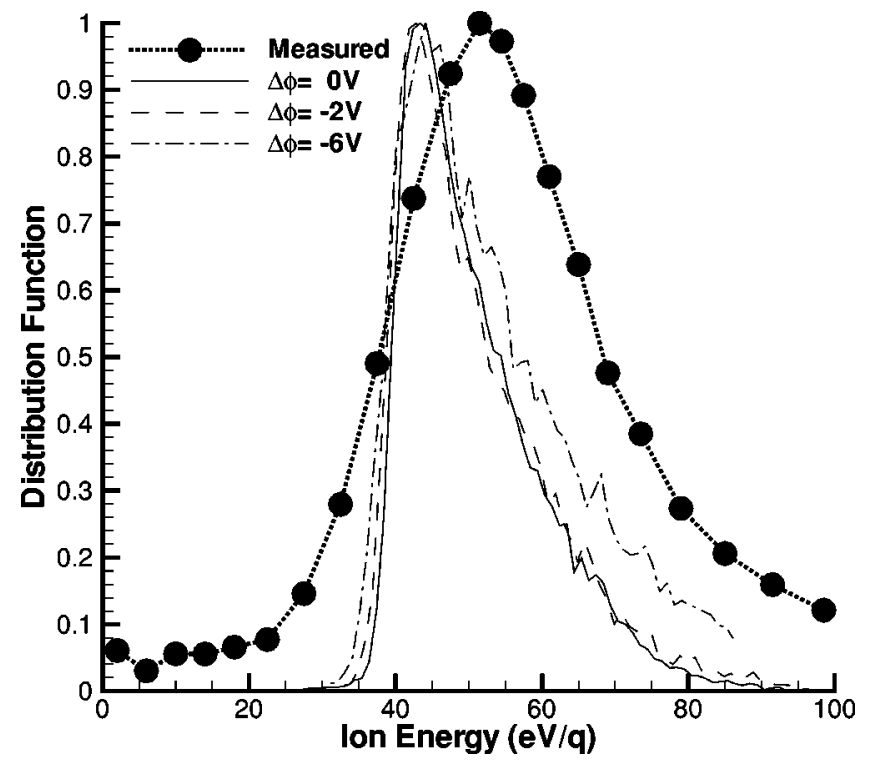

FIG. 24. Ion energy distribution at $21 \mathrm{~cm}$ from the hollow cathode exit for operating point C: Effect of varying initial plasma potential.

in all cases the simulation profiles are relatively independent of the initial temperature and under predict the measured data.

Figures 24 and 25 show the ion energy and current comparisons in which the initial plasma potential is varied $\left(T_{e}\right.$ $=2.5 \mathrm{eV}$ and standard transport coefficients are used for these cases). The shift of the IEDF corresponding to $\Delta \phi$ is not clearly seen here as it was for operating point A. However, as was the case for point $\mathrm{A}$, decreasing $\Delta \phi$ leads to a lower current profile. Finally, Figs. 26 and 27 show the effects on ion energy and current of varying the transport coefficients $\left(T_{e}=T_{i}=2.5 \mathrm{eV}\right.$ and $\Delta \phi=0 \mathrm{~V}$ for these cases). Increased values of $\nu_{e i}$ give the best agreement with the measured current profile, but these simulations lead to significant narrowing of the IEDF.

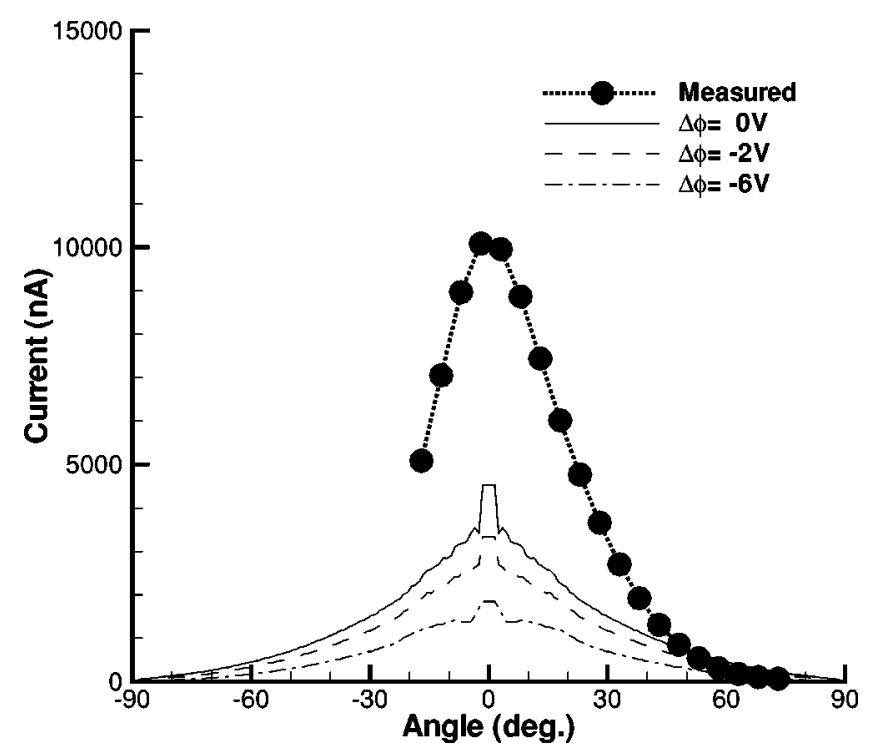

FIG. 25. Angular profile of ion current at $21 \mathrm{~cm}$ from the hollow cathode exit for operating point $\mathrm{C}$ : Effect of varying inital plasma potential.

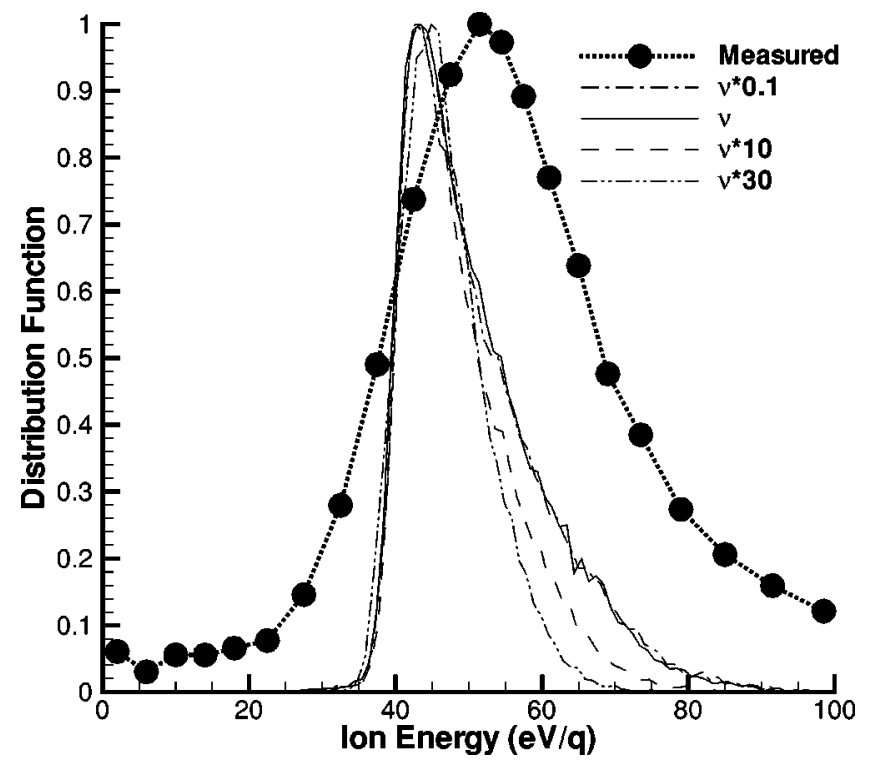

FIG. 26. Ion energy distribution at $21 \mathrm{~cm}$ from the hollow cathode exit for operating point $\mathrm{C}$ : Effect of varying transport coefficients.

In summary, operating point $C$ seems to involve a different physical regime from operating points A and B. Reasonable agreement with the measured data for both ion energy distribution and current is only obtained by using a very high initial electron and ion temperature $(4.0 \mathrm{eV})$ and increased value of $\nu_{e i}\left(\nu_{e i}^{*} 70\right)$. This suggests that either plasma turbulence is important in this case leading to decreased conductivities, or that some additional physical mechanism not included in the present model becomes important for these conditions.

\section{CONCLUSIONS}

A hybrid particle-fluid PIC-DSMC model was developed to model the flow from the orifice exit, through the keeper, into the plume of a xenon hollow cathode used for propellant

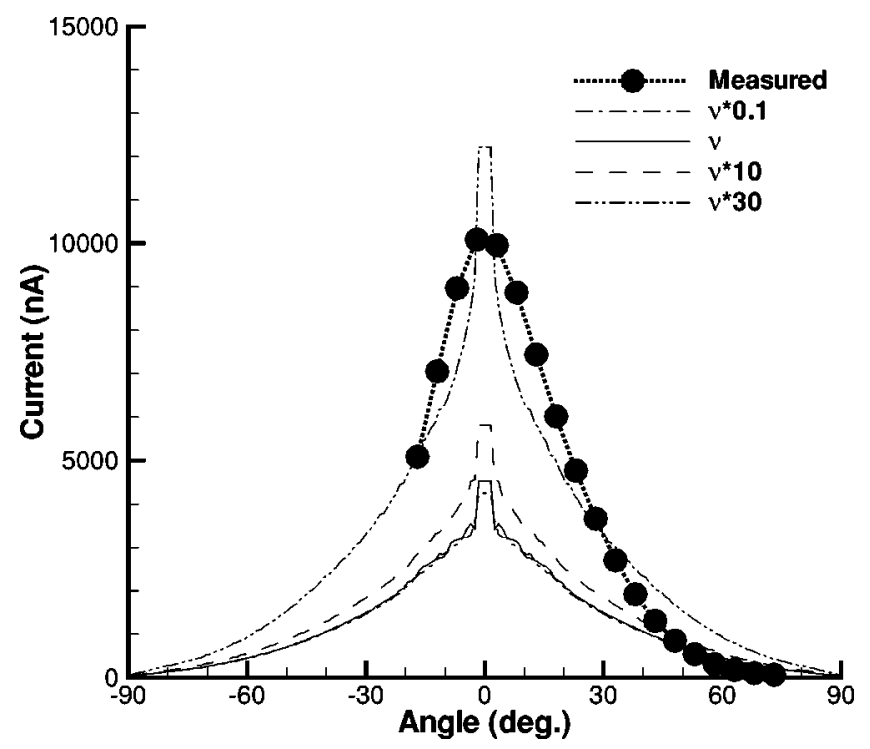

FIG. 27. Angular profile of ion current at $21 \mathrm{~cm}$ from the hollow cathode exit for operating point $\mathrm{C}$ : Effect of varying transport coefficients. 
ionization and beam neutralization in a spacecraft propulsion system. The model included a detailed fluid description of the electrons based on their continuity, momentum, and energy equations. The model was assessed by its application to three different operating points of the cathode for which experimental data exists. The sensitivity of the computed results to fundamental assumptions concerning the orifice exit conditions and the plasma transport properties was investigated. Increases in the exit electron and ion temperature led to broader ion energy distributions with an increased most probable energy. The high peak ion energies were generated in the simulations by the formation of a potential hill downstream of the keeper that is mainly formed by a plasma density gradient. By contrast, ion current profiles were relatively insensitive to the exit temperatures. Decreasing the orifice exit plasma potential also increased the most probable ion energy and led to significant decreases in the ion current. Decreased transport coefficients led to a decreased most probable ion energy and increased ion current.

Two of the cathode operating points (A and B) appeared to have similar behavior, for which the computer model provided excellent agreement with measurements of ion energy distribution, ion current, plasma potential, and electron temperature under reasonable orifice conditions and without changing the transport coefficients. Operating point $\mathrm{C}$ appeared to lie in a different physical regime where agreement between the simulation and measured data was only obtained by assuming high orifice exit electron and ion temperatures and reduced transport coefficients. It is concluded that this regime either involves significant plasma turbulence, leading to decreased conductivities, or that some physical mechanism missing from the present model has become important.

\section{ACKNOWLEDGMENTS}

I.D.B. gratefully acknowledges Dr. Matt Domonkos of NASA Glenn Research Center for use of his hollow cathode analysis code.

${ }^{1}$ I. Kameyama and P. J. Wilbur, AIAA 96-3208, 32nd AIAA/ASME/SAE/ ASEE Joint Propulsion Conference and Exhibit, Orlando, FL, 1996.

${ }^{2}$ P. M. Latham, A. J. Pearce, and R. A. Bond, IEPC 91-096, 22nd International Electric Propulsion Conference, Viareggio, Italy, 1991.

${ }^{3}$ V. J. Friedly and P. J. Wilbur, J. Propul. Power 8, 635 (1992).

${ }^{4}$ I. Kameyama and P. J. Wilbur, J. Propul. Power 16, 529 (2000).

${ }^{5}$ M. W. Crofton, J. Propul. Power 16, 157 (2000).

${ }^{6}$ I. Kameyama and P. J. Wilbur, ISTS Paper 98-a-2-17, 21st International Symposium on Space Technology and Science, Omiya, 1998.

${ }^{7}$ S. W. Patterson and D. G. Fearn, IEPC 99-125, 26th International Electric Propulsion Conference, Kitakyushu, Japan, 1999.

${ }^{8}$ G. Y. Yushkov, A. Anders, E. M. Oks, and I. G. Brown, J. Appl. Phys. 88, 5618 (2000)

${ }^{9}$ C. Wieckert, Phys. Fluids 30, 1810 (1987).

${ }^{10}$ G. J. Williams, T. B. Smith, M. T. Domonkos, A. D. Gallimore, and R. P. Drake, IEEE Trans. Plasma Sci. 28, 1664 (2000).

${ }^{11}$ M. T. Domonkos, Doctoral Thesis, Department of Aerospace Engineering, University of Michigan, September, 1999.

${ }^{12}$ C. K. Birdsall and A. B. Langdon, Plasma Physics Via Computer Simulation (Adam Hilger, Boston, 1991).

${ }^{13}$ G. A. Bird, Molecular Gas Dynamics and the Direct Simulation of Gas Flows (Oxford University Press, Oxford, 1994).

${ }^{14}$ D. E. Parks, M. J. Mandell, and I. Katz, J. Spacecr. Rockets 19, 354 (1982).

${ }^{15}$ J. D. Williams and P. J. Wilbur, J. Spacecr. Rockets 29, 820 (1992).

${ }^{16}$ M. Mitchner and C. H. Kruger, Partially Ionized Gases (Wiley, New York, 1973).

${ }^{17}$ E. Ahedo, P. Martinez-Cerezo, and M. Martinez-Sanchez, Phys. Plasmas 8, 3058 (2001).

${ }^{18}$ A. Dalgarno, M. R. C. McDowell, and A. Williams, Proc. R. Soc. 250, 411 (1958).

${ }^{19}$ S. H. Pullins, Y. Chiu, D. J. Levandier, and R. A. Dressler, AIAA 20000603, 38th AIAA Aerospace Sciences Meeting, Reno, 2000.

${ }^{20}$ S. Miller, D. J. Levandier, Y. Chiu, and R. A. Dressler, J. Appl. Phys. 91, 984 (2002).

${ }^{21}$ A. Von Engel, Ionized Gases (Oxford University Press, London, 1965). 